\title{
Think Tank Corner
}

\section{- Trend}

\section{Wachablösung an den Spitzen amerikanischer Think- Tanks}

Amerikanische Think-Tanks gelten gewöhnlich als „Regierungen im Wartestand“. Aufgrund des sogenannten „Drehtüreffekts “ wechseln zahlreiche Mitarbeiter der Denkfabriken in Regierungsjobs und viele ehemalige Regierungsmitarbeiter überwintern in Think-Tanks. Für das Führungspersonal der Think-Tanks gilt diese Regel indes nicht. Häufig amtieren die - bis vor kurzem zumeist männlichen - Direktoren über Jahre und sogar Jahrzehnte. So ist der Direkter der konservativen Heritage Foundation, Ed Feulner, seit 1977 im Amt, der Gründungsdirektor des libertären Cato Institute noch länger. Doch nun zeichnet sich in vielen etablierten Think-Tanks eine Wachablösung ab. Den Anfang machte bereits 2009 das American Enterprise Institute, wo Christopher DeMuth seinen Führungsjob nach über 20 Jahren abgab. Es folgten die RAND Corporation und jüngst das Obama-nahe Center for American Progress. Dort gab der seit 2003 an der Spitze stehende einstige Clinton-Stabschef und Gründungsdirektor John Podesta seinen Posten im Herbst 2011 ab. An seine Stelle trat mit Neera Tanden erstmals eine Frau. Auch drei weitere Think-Tanks aus dem links-liberalen Spektrum erhielten kürzlich weibliche Direktorinnen: die Century Foundation mit Janice Nittoli, das Urban Institute mit Sarah Rosen Wartell und das Roosevelt Institute mit Felicia Wong.

Kenner der amerikanischen Think-Tank-Szene glauben, dass es heute schwerer ist, einen Think-Tank in den USA zu leiten als früher. Gründe dafür sind, dass man mehr mit geringeren finanziellen Mitteln tun muss, dass die Konkurrenz größer geworden ist und dass das kommunikative Umfeld sich durch Blogs, soziale Medien sowie den rund-um-die-Uhr Nachrichtenzyklus fundamental verändert hat. Es ist für das einzelne Institut schwerer als früher, die geeigneten Zielgruppen ausfindig zu machen und dort Gehör zu finden. Was noch schwerer wiegt: Viele Geldgeber sind ungeduldig und wollen eine schnelle Wirkung ihres in einen Think-Tank investierten Geldes sehen. Dadurch fühlen sich Think-Tanks genötigt, plakativer, zuspitzender und politischer zu werden, anstatt sich auf längerfristige Politikanalyse und Vordenken zu konzentrieren.

\section{Debatte}

Interessengeleitete oder ungebundene wissenschaftliche Politikberatung? Eine Kontroverse

Schon mehrfach hat der Think-Tank-Corner der ZPB über Debatten in der wirtschaftspolitischen Ökonomik, über die Selbstreflexion wirtschaftspolitischer Berater sowie über Kontroversen innerhalb und um führende Wirtschaftsforschungsinstitute in Deutschland berichtet, etwa in den Heften 1 und 2/2010. An diese Berichterstattung wollen wir anknüpfen:
Die Debatte um das Selbstverständnis der wirtschaftspolitischen Beratung zog mittlerweile weitere Kreise: Am 4. Februar 2012 veröffentlichte die in Düsseldorf erscheinende Tageszeitung Handelsblatt einen Gastkommentar des ehemaligen Wirtschaftsweisen, Darmstädter Ökonomieprofessors und heute in einem gemeinsam mit Carsten Maschmeyer geführten privaten Beratungsunternehmen ${ }^{1}$ tätigten Bert Rürup mit dem Titel „Wenn Wissenschaftler Politiker beraten“.2 Darin weist Rürup zunächst auf die unterschiedlichen Funktionslogiken von Politik (Macht- und Sachlogik) und Wissenschaft (Suche nach logisch stimmigen und empirisch belastbaren Aussagen) hin. Bis dahin war der Gastkommentar nicht geeignet, Widerspruch zu ernten. Doch danach unterscheidet Rürup die Einflusskanäle Begutachtung und Beratung. Begutachtung, so Rürup, erfolge durch Bücher, Gutachten, Aufsätze und Interviews und wende sich sowohl an Entscheidungsträger als auch an die breitere Öffentlichkeit. Bei der Begutachtung urteilten Wissenschaftler nach bestem Wissen und Gewissen und empfehlen auf der Basis ihrer wissenschaftlich gewonnenen Überzeugungen. Auch an diesem Punkt erntet Rürup wenig Widerspruch. Kontrovers wird die Kolumne erst, als Rürup sich seinem Verständnis von wissenschaftlicher Beratung zuwendet. Diese habe und benötige immer einen spezifischen Adressaten wie z.B. eine Regierung, eine Partei/Fraktion oder einen Verband. „Das Ziel“, so schreibt Rürup, „sind Handlungsempfehlungen - inhaltlicher oder kommunikativer Natur -, und zwar unter Beachtung der Zielvorstellungen und der Entscheidungsrestriktionen des jeweiligen Adressaten. Letztendlich müsse ein Berater die Ziele des Beratenen akzeptieren und damit auch dessen Erfolg wollen.“ Diese „interessengeleitete“ Beratung, die sich an den Bedürfnissen eines konkreten Adressaten orientiere und nicht an der Regeln der „objektiven“ Begutachtung, sei in Deutschland - im Gegensatz zum angelsächsischen Raum oder auch Frankreich - als wissenschaftliche Politikberatung kaum akzeptiert, so das Mantra des ehemaligen Wirtschaftsweisen.

Dass Rürup mit seiner Einschätzung richtig lag, die adressaten- und interessengeleitete Beratung kollidiere mit dem in Deutschland von der Wissenschaft hochgehaltenen Postulat vermeintlich - werturteilsfreien und nur an der Wahrheit orientierten wissenschaftlichen Arbeitens, zeigte wenige Wochen später die deutliche Reaktion des Präsidenten des Deutschen Hochschulverbandes (DHV), Bernhard Kempen, im Hauptberuf Professor für öffentliches Recht und Völkerrecht an der Universität zu Köln, auf Rürups Zeitungsbeitrag. In seinem Vortrag zur Eröffnung des 62. Verbandstages des DHV, der Standesvertretung der deutschen Hochschullehrer, am 19. März 2012 in Hannover, der dem Thema „Unparteilichkeit der Wissenschaft“ gewidmet war, griff Kempen die Aus-

1 Beratungsgesellschaft für Alters- und Gesundheitsfürsorge.

2 Der Beitrag ist auf www.handelsblatt.com verfügbar [http://bit.ly/ zOgT8M]. 
sagen des „verehrten Kollegen Rürup“ zu wissenschaftlicher Politikberatung direkt an. ${ }^{3}$ Nach Auffassung Kempens ist es nicht falsch, sondern folgerichtig, Wissenschaftlern in der Rolle des "gebundenen Beraters" wenig institutionelle Wertschätzung entgegen zu bringen. „Nicht jede Politikberatung durch einen Wissenschaftler ist wissenschaftliche Politikberatung", so Kempen. Kempen - und vermutlich die Mehrheit der Verbandsmitglieder - wollen das Attribut „wissenschaftlich“ nur einer Politikberatung zugeordnet sehen, die ergebnisoffen und ungebunden ist. Dagegen betont der ehemalige Regierungsberater Rürup, dass der Wissenschaftler auch bereit sein muss, auf Zeit in die Rolle eines wissenschaftlichen Dienstleisters für einen spezifischen Adressaten zu wechseln, um Wirkung zu erzielen.

Bert Rürup ist nicht irgendwer in der wirtschaftspolitischen Beratung. Neben seinem derzeitigen Job in der Privatwirtschaft ist er der Vorsitzende des Kuratoriums eines der füh- renden Wirtschaftsforschungsinstitute in Deutschland, des Deutschen Instituts für Wirtschaftsforschung. Wie auch immer: Bert Rürup belebt sowohl die Theorie als auch die Praxis der wirtschaftspolitischen Beratung in Deutschland.

Martin Thunert, Heidelberg Center for American Studies, E-Mail:Martin.thunert@t-online.de

3 Siehe den Beitrag von Bernhard Kempen „Unparteilich, weil unparteiisch. Über die Rolle der Wissenschaft zwischen Politikberatung und dem Kampf um Drittmittel." in Forschung und Lehre, 5/2012, S. 372-373.

\section{Demokratieforschung}

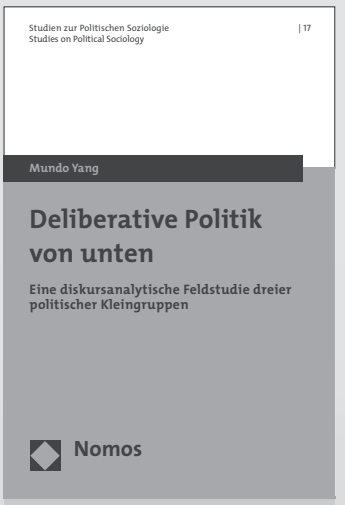

Deliberative Politik von unten

Eine diskursanalytische Feldstudie dreier politischer Kleingruppen

Von Mundo Yang

2012, 232 S., brosch., 39,- $€$

ISBN 978-3-8329-7247-9

(Studien zur Politischen Soziologie.

Studies on Political Sociology, Bd. 17)

www.nomos-shop.de/14400

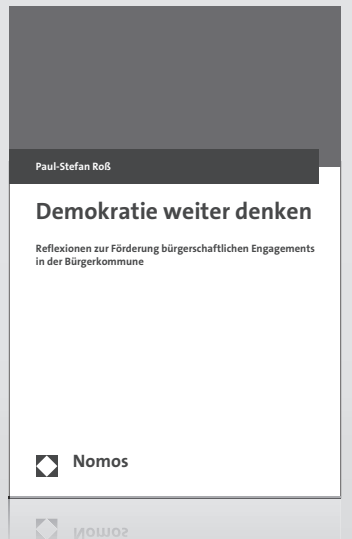

\section{Demokratie weiter denken}

Reflexionen zur Förderung bürgerschaftlichen Engagements in der Bürgerkommune

Von Paul-Stefan Roß

2012, 632 S., brosch., 49,- $€$

ISBN 978-3-8329-6470-2

www.nomos-shop.de/13474 